\title{
Pathopsychological Phenomena in Schizophrenia in the Context of Entropy Neuron-Glial Networks of the Brain
}

\author{
Rosman SV* \\ Physician of functional diagnostics of SBIH, Regional psychoneurological clinic, Russia
}

Submission: October 30, 2017; Published: November 15, 2017

*Corresponding author: Rosman SV, Physician of functional diagnostics of SBIH, Regional psycho neurological clinic, Tver, Russian Federation, Russia, Tel: +7-903-800-11-05; Email: seros2005@mail.ru

\begin{abstract}
The article attempted to search the connection between the main pathopsychological phenomena characteristic of schizophrenia and disruption of neuron-glial networks of the brain

Keywords: Neurophysiological basis of pathopsychological phenomena in schizophrenia; Entropy of the neuron-glial network of the brain; Dispersion of alpha-rhythm; Diagnosis of mental illness

Abbreviations: PD: Personality Disorder (F60.x ICD-10); BPD: Borderline Personality Disorder; BS: Burnout Syndrom; PPhT: Pedophilia in all Categories of Psychopathology; PPhH: Pedophilia is in the Category of Healthy; DSch: Debut of Paranoid Schizophrenia (F20.09x ICD-10); DAFCAR: Dispersion of Amplitude-Frequency Characteristics of the Alpha Rhythm; NGNB: Neuron-Glial Network of the Brain; HVT: Hyperventilation Test
\end{abstract}

\section{Introduction}

"The clinical picture of mental illness is the sum of psychopathological symptoms, syndromes and pathopsychological phenomena. Regarding the latter never had any doubts about the unreliability of the substrate and the local binding, or claims to their objectification. Thus, pathopsychological changes in patients, in particular patients with schizophrenia, has always been regarded as a mental (psychological) functioning is altered in disease conditions." This brilliant ring passage, drawn on one of the numerous medical sites, characterized the main reason why psychiatry is in deep crisis. In psychiatry, all agreed on the incomprehensibility of the causes of abnormal psychology mental illness, and it is carried out mainly on the degree of proximity of the descriptions of clinical manifestations and originality and philosophical studies of the phenomenon.

What pathopsychological phenomena we have to operate, considering a disease such as schizophrenia, which is undoubtedly of key importance to psychiatry [1].

A. First of all, this is "pathological polysemanticism." The background of our communication is united by a system of conventional symbols, i.e. concepts about which there is a kind of conditional "arrangement" - what it is and how it looks.
For example, if we say "table", then, despite all the differences associated with individual perception, the table is something on 4 legs with an even surface. The presence of his tail or eyes, bent into a spiral countertop is somehow not supposed. However, this is often what a schizophrenic table sees. Moreover, he can combine objects and events into one category according to a purely formal sign. To the question: "What is the difference between a cat and an apple" was the patient's response

"A cat eats meat, and an apple does not eat meat." At the same time, the semantic boundaries are so blurred that they sometimes completely disappear, and the patient loses all connection with the surrounding reality. He begins to arbitrarily apply the totality of facial expressions and gestures, phraseological constructs, which are the basis for communication of people in society. Until a certain moment, it is perceived by others as shocking, original, "dissent". Often this allows us to see some "genius" in nontrivial reasoning based on the "amalgam parallel", the distorted actualization of the insignificant properties of phenomena, giving them special significance.

However, all this colorful genius proves to be imaginary, has a point character. The gain from this is minimal, and the social loss is enormous. It has been proved that the 
orientation of schizophrenic patients on socially important signs, the recording and evaluation of which is a necessary condition for communication, is significantly deteriorating. They neglect common values, like the appearance of neatness, a manifestation of human qualities: love of others, diligence, compassion. In fact, they are unable to lead a normal life if they do not find compassionate understanding from family and friends, colleagues at work. So, the social-cognitive level of mental functioning of schizophrenics is characterized by the multivaluedness and uncertainty of perceived information.

B. Another generally recognized pathopsychological phenomenon in schizophrenic illness is referred to as "actualization of latent signs" or "superinclusion" [2]. This phenomenon concerns that part of cognitive activity, which in norm and pathology is closely related to the functioning of human memory. The process of processing information is controlled by the pattern recognition system. that in schizophrenia elective (selective) extraction of information from the mnemonic volume deteriorates. At the same time, the effect of intellectual activity qualitatively changes due to two processes, which can be designated as compensatory processes. Firstly, this is an extension of the range of information involved; secondly, their formalization or loss of probabilistic preference based on pragmatic significance. As a result, there is a tendency to update the almost insignificant (weak, latent) signs and aspects of objects, reducing the level of selectivity of information from past experience.

It is quite natural conclusion that the violation of the selectivity of data acquisition from memory is the central link in the changed structure of the functioning of the intellect in schizophrenia.

The explanation of this phenomenon makes it possible to clearly define pathopsychological features related to the definition of "schizophrenia" and designated as "ataxia", "schism", "discordance", etc. Deterioration of the actualization of information from past experience leads to a gradual semantic emasculation of the activity of the intellect with relative preservation of the structural side thinking (for example, the grammatical preservation of speech observed in many patients with its semantic incoherence).

C. The inadequate desire of many patients with schizophrenia to bring under any insignificant phenomenon, every "trifle", any "concept". As a result, there is a total "nonproductivity of the intellect" in the form of a long-winded "deficit". With careful evaluation it becomes clear that in the refraction of this pathopsychological phenomenon, a psychopathological symptom of resonance sounds (in a mild form - reasoning). Those "Fruitless wiserness" is not a reflection of the formal fallout of the intellect, but rather its new quality - the emergence of the paradigmatic orientation of schizophrenic cognitions.
For other constitutional and procedural pathopsychological phenomena, schizophrenic nosocertainty is not proven. There is no shortage of theories of the origin of schizophrenia. One list of them is quite impressive: neurotransmitter, serotonin, noradrenergic, dysontogenetic, psychoanalytic theory, constitutional and hereditary, the theory of autointoxication and autoimmunization, infectious, cognitive. For many decades, mental disorders are perceived as "malicious dysfunction", not correlated with violations in neuronal mechanisms, and in this connection psychiatry experienced a sharp decline, which resulted in a need to change the paradigm of psychiatric nosology.

In 2008, the Research on Domain Research (RDoC) project, initiated by the American National Institute of Mental Health (NIMH), was published [3]. RDoC differs from the study of syndromes using the dimensional approach. Instead of starting with diagnoses based on a clinical picture, and then connecting them with internal mechanisms [4], RDoC begins with dysfunctional mechanisms, moving from them to clinical symptoms. To some extent, this step is dictated by the desire to fill the gap between neuroscience and the study of mental disorders, emphasis was placed on communication with the neural network, not obliging to adopt a reductionist philosophy; on the contrary, they provide various important proofs of observation of different systems.

The main argument of opponents of the consideration of mental processes from the standpoint of dimensionality is the accusation of reductionism - the reduction of an inconceivably complex mental activity to "simplified" mechanical and biological models. Moreover, it does not take into account the fact that not only because Nature has "nailed" this entire complex structure called the brain. And then the suspicion that the furious protection of the "divine incomprehensibility" of mental processes is based on an elementary ignorance of the foundations of mathematics, cybernetics, informatics and neurophysiology begins to creep in. How can the pathopsychological phenomena of schizophrenia hypothetically look from the standpoint of information interaction of afferent signals and a neural-glial network?

Pathological polysemanticism, from the standpoint of computer science, is a typical error in the coding of a signal. During the Second World War, the Germans invented the Enigma encryption machine. The essence of it was that the keyboard typed text letters, which when you move the keys with the help of the internal gear mechanism "Enigma" were transformed into a digital code. At the same time, while maintaining the invariance of gear ratios, each letter was matched by a certain combination of numbers. The combination of numbers was transmitted to the recipient, and he, with the help of a reverse mechanism, deciphered the message. The basic condition for the correct perception of information was the absolute identity of the gear mechanisms at the transmitting and receiving sides. And now 
imagine that with the same settings, the transmitting machine broke a tooth on one of the gears, and it began to "scroll", changing the gear ratio. The modified information will be transmitted, which on the receiving side will either not be decrypted, or it will be decrypted incorrectly. By the way, comprehending the secrets of the Enigma, the outstanding English cybernetist A. Turing first put forward the idea of self-organization of chaotic structures, which underlies the fractal neurophysiological model of brain activity [5].

Approximately the same thing happens in NGNB, which is affected by the schizophrenic process. Numerous DAFCAR studies confirmed by many clinicians have proved that schizophrenia is characterized by the phenomenon of functional hypofrontal function caused by the functional inconsistency of a part of NGNB cells in the frontal lobes and, as a consequence, inability to adequately internalize - transforming external real actions, properties of objects, social forms of communication into stable socio- acceptable patterns of behavior. Is it really impossible to assume that these coincidences are not accidental, especially since they are constantly encountered? In this connection, it can be assumed that both dispersion cartograms and DAFCAR indices can objectively reflect the presence of a schizophrenic process [6,7].

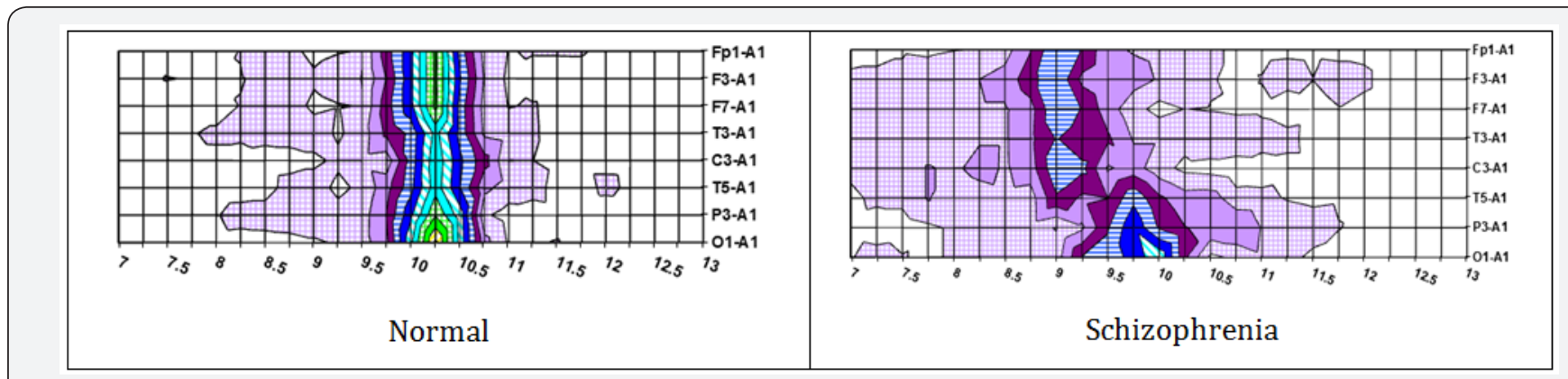

Figure 1: Averaged dispersion cartograms of alpha rhythm in norm and in schizophrenia.

Thus, the cause of pathologic polysemanticism is functional hypofrontality, caused by the disorganization of the NGNB work in the frontal lobes due to the dissociation of the properties of its constituent nerve cells. All the variety of clinical manifestations of polysemanticism is a consequence of the degree and the current prevalence of this disorganization (Figure 1). Of course, there is the question of reductionism, that supercomplex movements of the human soul are trying to explain "by some kind of" variances in the amplitude-frequency characteristics of the activity of neurons. In fact, all this is simple, if you do not know anything about it. In fact, this is a manifestation of the fundamental processes of self-organization of chaotic systems under the influence of ordered information flows. This is surprising in its results the ability to change the functional structure of NGNB with the repeated presentation of an afferent stimulus, in which the efferent response of everything is compared with the Images Existing In Memory. That's why no image storage has been found so far, although, according to the estimated estimates, the amount of information should be measured in yottabytes. Therefore, it is easier to assume that memory, in the form we imagine it, does not exist [8].

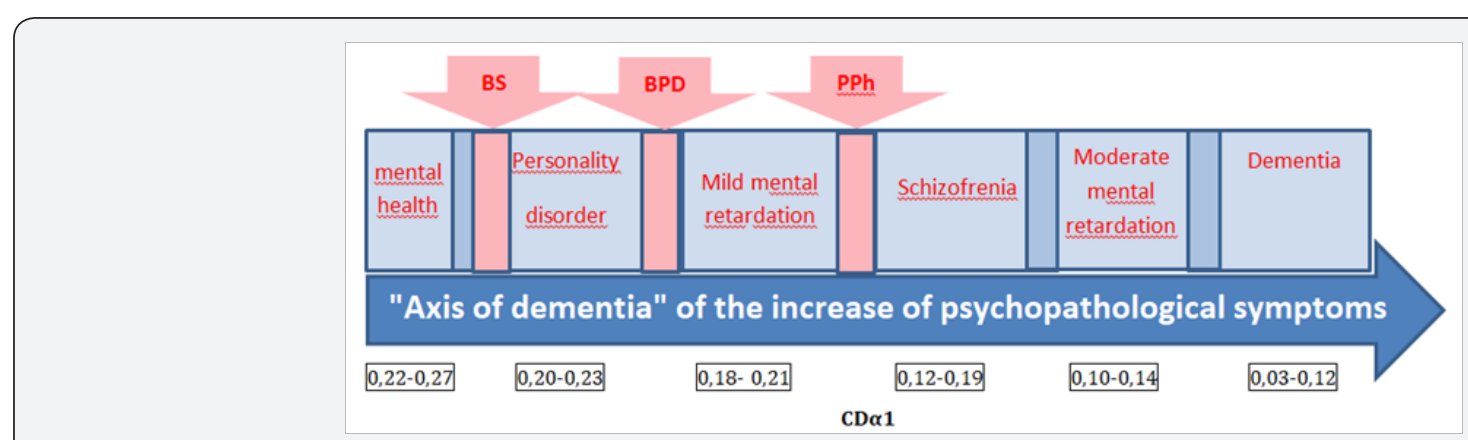

Figure 2: Place pedophilia on "axis of dementia "in the framework of the theory of "a single psychotic". BS - burnout, BPD - borderline personality disorder, $\mathrm{PPh}$ - pedophilia.

That is why the deterioration in elective (selective) extraction of information from the mental volume due to schizophrenia is not due to the fact that the brain is at the same time mistaken by the memory cell, but because it is destroyed by the NGNB. Thus, all these numerous pathopsychological phenomena in schizophrenia are explained by the disorganization of NHSGM in the frontal lobes. But schizophrenia is only part of the general problem of pathopsychology. In a number of articles in the DAFCAR cycle, an incredibly important question was raised about the problems of the formation of acceptable stereotypes 
of behavior in humans and the possibilities of diagnosing these disorders. In a number of articles on violations of entropy NGNB with borderline mental disorders [8] in violent criminals [9], pedophiles [10], with the "burnout syndrome" [11], the formation of alcohol quite clearly the general trend "embedding" of these states in the concept of the "single psychosis" theory. All of them are, as it were, representations of the schizophrenic process, a state of incomplete completion of the functional disorganization of NGNB in the frontal lobes. Is it accidental? (Figure 2).

Undoubtedly, all this tissue is extremely thin and requires the most careful study with the development of fairly objective criteria for the severity of disorders, the possibility of adapting the patient to the revealed disturbances in the surrounding world [12]. Of course, it is unacceptable to label a crazy person with hospitalization for everyone who has an alpharhythm dispersion. But it is impossible not to pass by this fact indifferently, especially if the lives and destinies of the surrounding people depend on this person.

In addition [13], it is quite obvious that DAFCAR is one of the first swallows in a number of other studies, perhaps more informative. However, the essence of them for a long time will be practically unchanged - the identification of the degree of functional disorganization of NGNB.

\section{References}

1. American Psychiatric Association Committee (1980) on Nomenclature and Statistics. Diagnostic and Statistical Manual of Mental Disorders (DSM-III). American Psychiatric Association, Washington DC, USA, pp. 494.
2. Andreasen NC (1986) Scale for the Assessment of Thought, Language, and Communication. (TLC)/N.C. Andreasen//Schizophr Bull 12: pp. 473-482.

3. Brüne M (2005) Theory of Mind in Schizophrenia: A Review of the Literature. Schizophr Bull 31(1): 21-42.

4. Rosman SV,Kurakhina OB (2017) Violent Crime in the Context of Entropy Neuron-Glial Networks of the Brain. Glob J Add \& Rehab Med 2(5).

5. Rosman SV, Maximova NE (2017) Paroxysmal-Progredient Paranoid Schizophrenia in the Context of Entropy Neuron-Glial Networks of the Brain. Glob J Add \& Rehab Med 4(1).

6. Rosman SV (2017) The Theoretical Foundations of Dispersion of Amplitude-Frequency Characteristics of the Alpha Rhythm of the EEG. Glob J Add \& Rehab Med 2(3).

7. Rosman SV (2017) The Use of Analysis of Variance of the Alpha Rhythm of the EEG in the Study of the Pathogenesis of Alcoholism and the Causes of Alcoholic Deliria. Glob J Add \& Rehab Med 2(1).

8. Rosman SV (2017) Borderline Personality Disorder in the Context of Entropy Neuron-Glial Networks of the Brain. Glob J Add \& Rehab Med 2(4).

9. Rosman SV, Filonov SM (2017) Cognitive Impairment in Oncology Practice in the Context of Entropy Neuron-glial Network of the Brane. Glob J Add \& Rehab Med 2(4).

10. Rosman SV (2017) Burnout Syndrome in the Context of Entropy Neuron-Glial Networks of the Brain. Glob J Add \& Rehab Med 3(3).

11. Rosman SV (2017) Pedophilia in the Context of Entropy Neuron Glial Networks of the Brain. Glob J Add \& Rehab Med 4(2).

12. Rosman SV (2017) The Debut of Schizophrenia in the Context of Entropy Neuron-Glial Network of the Brane. Glob J Add \& Rehab Med $3(4)$.

13. Sanislow Charles A (2016) Updating the Research Domain Criteria. World Psychiatry 15(3): 222-223.

\section{Your next submission with Juniper Publishers} will reach you the below assets

- Quality Editorial service

- Swift Peer Review

- Reprints availability

- E-prints Service

- Manuscript Podcast for convenient understanding

- Global attainment for your research

- Manuscript accessibility in different formats

( Pdf, E-pub, Full Text, Audio)

- Unceasing customer service

Track the below URL for one-step submission https://juniperpublishers.com/online-submission.php 